\title{
LDPC Code Design for OFDM Channel: Graph Connectivity and Information Bits Positioning
}

\author{
Alexandre de Baynast, Ashutosh Sabharwal, Behnaam Aazhang \\ Electrical and Computer Engineering Department \\ Rice University \\ Houston, Texas 77005-1892 \\ Email: debaynas, ashu, aaz @rice.edu
}

\begin{abstract}
We present an optimized channel coding scheme for OFDM transmission. In the future wireless standards such IEEE 802.11n, frame error rates (FER) as low as 0.0001 are targeting. Irregular LDPC codes have excellent performance at moderate computational complexity: they are strong candidates for channel coding in such systems. In the context of an OFDM transmission, the authors in [Mannoni, 2002] proposed to optimize the graph connectivity of the irregular LDPC code accordingly to the channel spectrum. However, their codes did not have good performance for a FER range of $0.001-0.0001$. In this paper, we propose to optimize the LDPC code in two steps: first, we optimize the graph connectivity in order to get a minimum operational average SNR and therefore excellent FER; then we optimize the information bits placement accordingly to the channel. By simulation, we show that our approach provides substantial performance gain in term of FER over the existing methods (1 dB or more for FER equal to $\left.10^{-4}\right)$.
\end{abstract}

\section{INTRODUCTION}

One of the ambitious design goals of future wireless systems, including 4G, IEEE $802.11 \mathrm{n} / 802.16$ standards, is to reliably provide very high data rate transmission in hostile environments: hundred(s) of $\mathrm{Mb} / \mathrm{s}$ or more for downlink transmission with a low frame error rate (FER), typically less than $5.10^{-4}$ [5]. Therefore, efficient equalizers and decoders are required in order to mitigate inter-symbol interference (ISI) and residual interference, respectively. OFDM modulation are particularly suited for transmissions over multipath channels, [8]. An OFDM system transforms the frequency selective channel into a set of narrowband Gaussian orthogonal subchannels. Since the frequency selectivity implies that some subbands are strongly weakened, a powerful receiver is needed. Several methods such as power allocation [1] or channel coding [9] have been used. In this paper, we only consider the second category also called Coded OFDM (COFDM). Among the different existing codes, Low Density Parity-Check (LDPC) present a great interest for COFDM transmission, as shown for instance in [3]. Much attention is being given to LDPC codes due to their excellent performance and the simplicity of iterative decoding making high throughput possible [2]. It has been shown in [4], that for an OFDM transmission, the optimization of the LDPC code accordingly with the channel spectrum provides large performance gain with no reduction of

\footnotetext{
${ }^{1}$ This work was supported in part by Nokia Corporation, Texas Instruments Inc., and by NSF under grants ANI-9979465, EIA-0224458, and EIA-0321266.
}

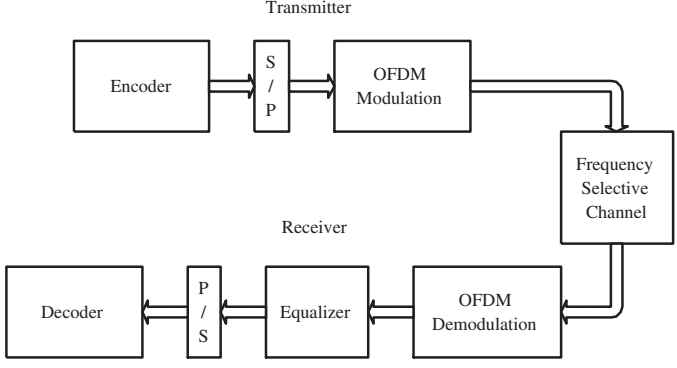

Fig. 1. LDPC-coded OFDM system.

the spectral efficiency. However, theses codes do not exhibit excellent FER. In this paper, in order to achieve FER below $5.10^{-4}$, we propose an alternate LDPC optimization for OFDM channel in two steps: first, we optimize the code through density evolution algorithm (DEA). Since DEA works in an asymptotic regime, i.e when the whole codeword is perfectly decoded, there is no mean to optimize simultaneously the information bits positioning. Then, in the second step, we determine the optimal placement of the information bits which minimizes the probability of error.

The paper is organized as follows: first, we describe the transmission model that we consider. In the second part, the problem of LDPC code design for OFDM is addressed. Next, the proposed optimization algorithm is presented. Some simulations results which validate our approach are shown in the part V. Section VI contains the conclusions.

\section{SySTEM DESCRIPTION OF LDPC CODED OFDM}

We consider an LDPC-coded OFDM system with $N_{c}$ subcarriers, signaling through frequency-selective channel. The communication system is illustrated in Fig. 1.

A block of $k$ bits of information data is encoded by a rate $r=k / n$ LDPC code. The coded $n$ bits are modulated by quadrature amplitude modulation (QAM) constellation of size $q$ into a block of $n / \log _{2}(q)$ QAM symbols. During each OFDM slot, $N_{c}$ out of the total $n / \log _{2}(q)$ QAM symbols are transmitted from $N_{c}$ OFDM subcarriers.

We consider a quasi-static block fading model for the studied coded OFDM modulation. It is assumed that the fading channels remain static during each OFDM slot but slightly vary from 


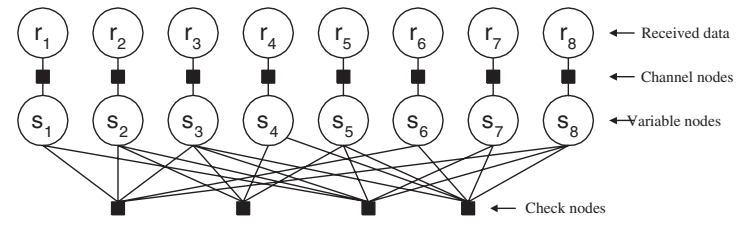

Fig. 2. Factor Graph Representation for an small irregular LDPC code.

one OFDM slot to another. Assuming proper cyclic insertion and sampling, the OFDM system with $N_{c}$ subcarriers decouples the frequency-selective channel into $N_{c}$ correlated flat-fading channels with the following input-output relation:

$$
r_{i}=h_{i} s_{i}+n_{i}, i=1, \ldots, N_{c},
$$

where $h_{i}$ is a complex channel spectrum coefficient. $s_{i}$ and $r_{i}$ are, respectively, the transmitted signal and the received signal at the $i$-th subcarrier; $n_{i}$ is the i.i.d. zero-mean additive noise with a variance $\sigma^{2}$.

Note that the generalization to a system with multiple transmit and receive antennas with full multiplexing scheme and linear equalization is straightforward. In that case, the coefficient $h_{i}$ would correspond to the combined channel+equalizer frequency domain response and we have $N_{t}$ parallel data streams, with $N_{t}$ the number of transmit antennas.

\section{ASYMPTOTIC PERFORMANCE OF THE IRREGULAR LDPC CODES}

\section{A. LDPC Decoding: Belief Propagation on Factor Graph}

As well as the Turbo-codes, irregular LDPC codes can achieve reliable transmission for a signal-to-noise ratio extremely close to the Shannon limit on the AWGN channel [6]. The LDPC codes can be represented by a bipartite graph that consists of two types of nodes: the variable nodes and the check nodes as shown on Fig. 2. Each code bit is a variable node, whereas each parity check equation represents a check node. An edge in the graph is placed between variable node $i$ and check node $j$ if there is a one in position $(i, j)$ in the parity-check matrix. Irregular LDPC codes are specified by two polynomials $\lambda(x)=\sum_{i=2}^{d_{c}} \lambda_{i} x^{i-1}$ and $\rho(x)=\sum_{i=2}^{d_{c}} \rho_{i} x^{i-1}$ where $\lambda_{i}$ is the fraction of edges in the bipartite graph that are connected to the variable nodes of degree $i$ and $\rho_{i}$ is the fraction of edges that are connected to check nodes of degree $i ; d_{c}$ and $d_{r}$ represent the maximal variable and check nodes connection degree, respectively.

The Belief Propagation (BP) algorithm is used to decode the LDPC codes [6] iteratively. Under the BP algorithm, variable nodes and check nodes exchange messages iteratively. It is convenient to use log-likelihood ratios (LLRs) as messages. Let $v$ and $u$ denote the output message of a variable and check node, respectively. Under the sum-product decoding, the message $v$ is equal to the sum of all incoming LLRs; i.e.:

$$
v=\sum_{l=0}^{i-1} u_{l}
$$

where $u_{l}, l=1, \ldots, i-1$, are the incoming LLRs from the neighbors of the variable node save the check node that gets the message $v ; i$ is the degree associated with the variable node and $u_{0}$ is the observed LLR of the output bit associated with the variable node.

The message update rule for check nodes can be expressed as follows:

$$
\tanh \frac{u}{2}=\prod_{l=1}^{i-1} \tanh \frac{v_{l}}{2}
$$

where $v_{l}, l=1, \ldots, j-1$, are the incoming LLRs from $j-$ 1 neighbors of a check node, and $u$ is the message sent to the remaining neighbor. After few iterations, the tentative A Posteriori Probability ratio for each variable node is equal to $v+u_{i}$.

In [7], the authors design low-density parity-check (LDPC) codes that perform at rates extremely close to the Shannon capacity. The codes are built from highly irregular factor graphs with carefully chosen degree patterns on both sides.

Besides their near Shannon limit performance, irregular LDPC codes have an important intrinsic property due to their irregularity: the higher the degree of a variable node, the more robust to the noise is the associated bit. In an OFDM transmission, the signal to noise ratio per subcarrier may significantly vary from one bit of the codeword to another. To provide a good reliability, the bits must be protected accordingly with the their relative SNR. Therefore, the position of connection degree of the variable node is an important parameter. Recently, this property has been exploited in [4]. Since the optimization of the LDPC codes is done by minimizing the probability of error, the optimized code does not have good performance in term of FER below the targeting probability of error. In the next section, we propose a LDPC code optimization in two steps in order to achieve low FER.

\section{B. Density Evolution under Gaussian Approximation for OFDM channel}

Assuming that the all-zero codeword is sent $\left(s_{i}=1, \forall i\right)$, the channel log-likehood ratio (LLR) are equal to:

$$
u_{0, i}=\log \left(\frac{p\left(r_{i} \mid s_{i}=1\right)}{p\left(r_{i} \mid s_{i}=-1\right)}\right)=\frac{2 h_{i}}{\sigma^{2}}\left(h_{i}+n_{i}\right)
$$

Therefore, the channel LLR messages $u_{0, i}$ are Gaussian with mean $m_{u 0, i}\left(h_{i}\right)=2 h_{i}^{2} / \sigma^{2}$ and variance $\sigma_{0, i}^{2}\left(h_{i}\right)=$ $2 m_{u 0, i}\left(h_{i}\right)$. As the variance is twice the mean and that for all iterations thanks to the symmetry condition [6], the message evolution can be characterized by the mean $m_{u 0, i}\left(h_{i}\right)$ only. In a real system, SNRs are different between the subcarriers (or equivalently for every variable node) and therefore the means $m_{u 0, i}\left(h_{i}\right)$ are different for each $i$ as well. In order to drastically reduce the number of parameters $m_{u 0, i}$, we use the same approximation that is proposed in [4]. The original channel spectrum shown on Fig. 3, is approximated by few coefficients. Indeed, in each sub-band $j, j=1, \ldots, J$, the fading coefficients are assumed to be equal to a constant denoted $\bar{h}_{j}$. In practice, $\bar{h}_{j}$ is the average SNR inside each sub-band. 


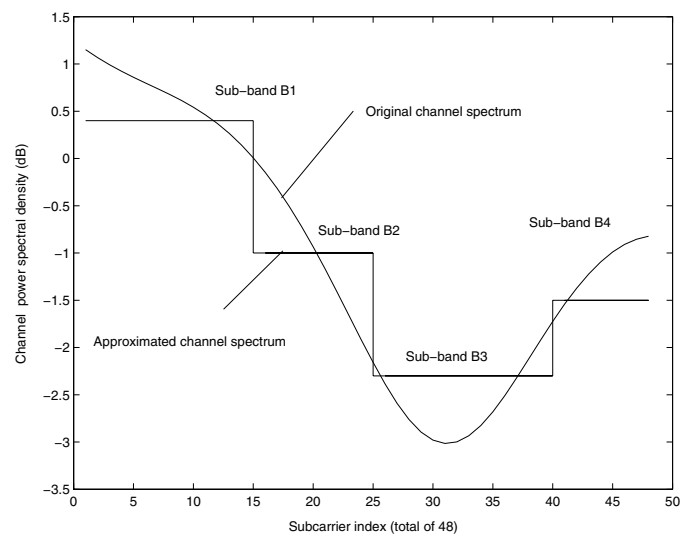

Fig. 3. Typical Channel Spectrum in an IEEE802.11n system. A 48 subcarriers system is considered. The SNRs for each subcarrier are calculated from the channels using the ITU model.

Thanks to this simplification, we are able now to perform the Density Evolution under Gaussian approximation for OFDM channels. Suppose that we have $J$ sub-bands. In every sub-band $j=1, \ldots, J$, the coefficients $\lambda_{i, j}, i=1, \ldots, d_{v}, j=1, \ldots, J$ correspond to the proportion of edges with a connection degree of $i$. Assuming flat fading over all subcarriers inside the $j$-th sub-band, the pdf of the channel LLRs is Gaussian with mean $m_{u 0, j}=2 \bar{h}_{j}^{2} / \sigma^{2}$ and variance $\sigma_{0, j}^{2}=2 m_{u 0, j}$.

We denote the means of the messages $u$ and $v$ (Eqs. 3 and 2) by $m_{u}^{(l)}$ and $m_{v}^{(l)}$, respectively at the iteration $l$. Then, for any sub-band $j, j=1, \ldots, J$, by taking the expectation in both sides in Eq. 2, the mean $m_{v, i, j}^{(l)}$ of the output message of a variable node with degree $i$ at the iteration $l$ is given by

$$
m_{v, i, j}^{(l)}=m_{u 0, j}+(i-1) m_{u}^{(l-1)}
$$

Since the output message of a variable node is characterized by its mean, which is the sum of the means of the incoming densities, we only need to keep the mean of the Gaussian mixture from check nodes to variable nodes ([7]). Therefore, by taking expectation in Eq. 3, it has been shown in [6] that the mean $m_{u}^{(l)}$ of the output message is given by:

$$
m_{u}^{(l)}=\sum_{k=2}^{d_{c}} \rho_{k} \phi^{-1}\left(1-\left[1-\sum_{i=2}^{d_{v}} \sum_{j=1}^{J} \lambda_{i, j} \phi\left(m_{v, i, j}^{(l)}\right)\right]^{k-1}\right)
$$

where the function $\phi(x)$ is equal to 1 $\frac{1}{\sqrt{4 \pi x}} \int_{-\infty}^{+\infty} \tanh \frac{u}{2} \exp \left(-\frac{(u-x)^{2}}{4 x}\right) d u, \forall x, x>0$ ([6]).

By combining Eqs. 5 and 6, we are able to track the evolution of $m_{u}^{(l)}$ through the iterative decoding process for an OFDMbased system. The minimal operational SNR $\mathrm{SNR}_{\min }^{*}$ can be computed as the minimum SNR for which the mean $m_{u}^{(l)} \rightarrow$ $+\infty$.

\section{OPTIMIZATION ALGORITHM OF THE CODE PROFILE}

The procedure for computing the minimum operational average SNR for a given degree profile $(\lambda(x), \rho(x))$ is used in conjunction with an optimization procedure to design optimal
LDPC code for OFDM channel. The idea is to find optimal $\lambda(x)$ and $\rho(x)$ such that the $\mathrm{SNR}_{\text {min }}^{*}$ is minimized. Since this criterion is achieved for a zero-error probability, the optimized code has very good performance in term of FER. However, the positioning of the information/redundancy bits can not be considered in this approach. For binary or AWGN channels, optimization of the information bits placement gives minor gains as shown in [4]. Nevertheless, for an OFDM transmission as noticed in [4], significant gain in term of Bit Error rate (BER) and FER are expected. Then, we propose the following 2-steps strategy:

1) Optimization of the degree distributions $\lambda(x)$ and $\rho(x)$ such that the $\mathrm{SNR}_{\text {min }}^{*}$ is minimized. We use exactly the approach advocated by Chung in [7].

2) Minimization of the probability of error for the values of $\lambda(x)$ and $\rho(x)$ determined in the first step.

We choose the information bits placement which minimizes the probability of error given by ([7]):

$$
P_{e}^{(l)}=\frac{1}{\sum_{i, j} \lambda_{i, j} / i} \sum_{i, j \in \operatorname{Inf}} \lambda_{i, j} / i Q\left(\sqrt{\frac{m_{u 0, j}+i m_{u}^{(l)}}{2}}\right)
$$

where $Q($.$) is the Q-Marcum function. The set Inf cor-$ responds to the fraction $\lambda_{i, j}$ of connection degree $i$ in the $j$-th sub-band dedicated to the information bits. The selection of the optimal set for the information bits is a non trivial problem. It can efficiently be solved by using density differentiation [4].

Tab. I gives the distribution pairs $(\lambda(x), \rho(x))$ we optimized for the channel shown on Fig. 3 for rate of $1 / 2$ with a maximal degree for the variable nodes equal to 10 . The channel spectrum is approximated by four sub-bands denoted B1, B2, B3 and B4. Their respective normalized average power are $2.9948 \mathrm{~dB}$, $0.3403 \mathrm{~dB},-4.3792 \mathrm{~dB}$ and $-2.5120 \mathrm{~dB}$ (the overall power is unitary after normalization). We observe that the connection degree is inversely proportional to the SNR in the sub-bands. This confirms the fact that a bit which is involved in a large number of parity-check equations is better protected against fading than those bits which are connected to a small number of check nodes. Moreover, the threshold for this code is equal to $4.0392 \mathrm{~dB}$. In order to evaluate the gain that we obtain by adjusting the connection degree distribution to the channel fading, we computed also the threshold for the same profile with a random interleaver (the profile is then the same for each sub-band). The threshold for a random interleaver is $6.2207 \mathrm{~dB}$ i.e. more than $2 \mathrm{~dB}$ loss with the optimal scheme we proposed.

\section{Simulation Results}

In the previous section, the threshold values predict the asymptotic performance as the block length of the LDPC codes approaches infinity. We are also interested in the performance of the optimized irregular LDPC codes when moderate block size is considered. In the following, we consider the optimized 1/2-rate LDPC code at a block size $n$ of 1152 bits compatible 


\begin{tabular}{|c|c|c|c|c|}
\hline $\begin{array}{c}\text { sub-band } \\
\text { average power }\end{array}$ & $\begin{array}{c}\mathrm{B} 1 \\
2.9948 \mathrm{~dB}\end{array}$ & $\begin{array}{c}\mathrm{B} 2 \\
0.3403 \mathrm{~dB}\end{array}$ & $\begin{array}{c}\mathrm{B} 3 \\
-4.3792 \mathrm{~dB}\end{array}$ & $\begin{array}{c}\mathrm{B} 4 \\
-2.5120 \mathrm{~dB}\end{array}$ \\
\hline \hline$\lambda_{2,1: 4}$ & 0.0833 & 0.1530 & 0.0000 & 0.0000 \\
\hline$\lambda_{3,1: 4}$ & 0.0000 & 0.0415 & 0.0000 & 0.2002 \\
\hline$\lambda_{4,1: 4}$ & 0.0000 & 0.0000 & 0.0000 & 0.0000 \\
\hline$\lambda_{5,1: 4}$ & 0.0000 & 0.0000 & 0.0000 & 0.0000 \\
\hline$\lambda_{6,1: 4}$ & 0.0000 & 0.0000 & 0.0000 & 0.0000 \\
\hline$\lambda_{7,1: 4}$ & 0.0000 & 0.0000 & 0.0000 & 0.0000 \\
\hline$\lambda_{8,1: 4}$ & 0.0000 & 0.0000 & 0.0000 & 0.0000 \\
\hline$\lambda_{9,1: 4}$ & 0.0000 & 0.0000 & 0.0000 & 0.0000 \\
\hline$\lambda_{10,1: 4}$ & 0.0000 & 0.0000 & 0.4167 & 0.1054 \\
\hline \hline$\rho_{7}$ & \multicolumn{5}{|c|}{0.0254} \\
\hline$\rho_{8}$ & \multicolumn{5}{|c|}{0.07456} \\
\hline \hline$E_{b} / N_{0}$ & \multicolumn{5}{|c|}{$\mathrm{dB}$} \\
\hline
\end{tabular}

TABLE I

GOOD DEGREE DISTRIBUTION PAIRS OF RATE-1/2 FOR THE OFDM CHANNEL DEPICTED IN FIG. 3 WITH CONTRAINT ON THE MAXIMAL LEFT DEGREES $d_{v}=10$.

with the standard IEEE 802.16 [5]. It is generated according to the profile given in Tab. I. We compare four following schemes:

1) the decoder based on the optimized code from Tab. I (solid line with marker ' ${ }^{*}$ '). Additionally, information bits placement is choosen in order to minimize the probability of error given in Eq. 7.

2) the decoder based on the optimal code determined in the previous section (solid line with marker ' $\Delta$ '). Information bits are mapped to the variable nodes with the largest degrees. This strategy minimizes the probability of error for higher SNR.

3) The approach proposed in [4] (solid line with marker ' $\square$ '). In their approach, they minimize the probability of error; therefore, the redundancy bits are mapped to the subcarriers with the weakest SNRs. This strategy does not give the best convergence threshold and penalizes the FER at high SNR.

4) The variable nodes with the highest connectivity degree are mapped to the subcarriers with the largest SNRs (solid line with marker 'o').

Fig. 4 illustrates the performance for the OFDM channel shown on Fig. 3. Fading coefficients of this channel are given in Tab. I. The code rate is $1 / 2$ and the codeword length is fixed to 1152 for all plotted schemes. It corresponds to 12 consecutive OFDM symbols where 48 subcarriers are used for the data transmission. Clearly, the code for which the profile minimizes the threshold achieves the best performance for low targeting FERs. Code based on the minimization of the probability of error ([4]) has also good performance and outperforms the reverse approach proposed in the case 4 . However, for small FER (less than $4.10^{-3}$ ), the code that we propose in this paper outperforms [4]. For a FER of $5 \cdot 10^{-4}$, the gap is significant (1 $\mathrm{dB})$. We can conclude that our coding strategy is well suited for the IEEE $802.11 \mathrm{n}$ standard. Little improvement can be obtained by optimizing the placement of the information bits. However, with this strategy, we can not fill the gap with [4] for moderate FER $(>0.01)$.

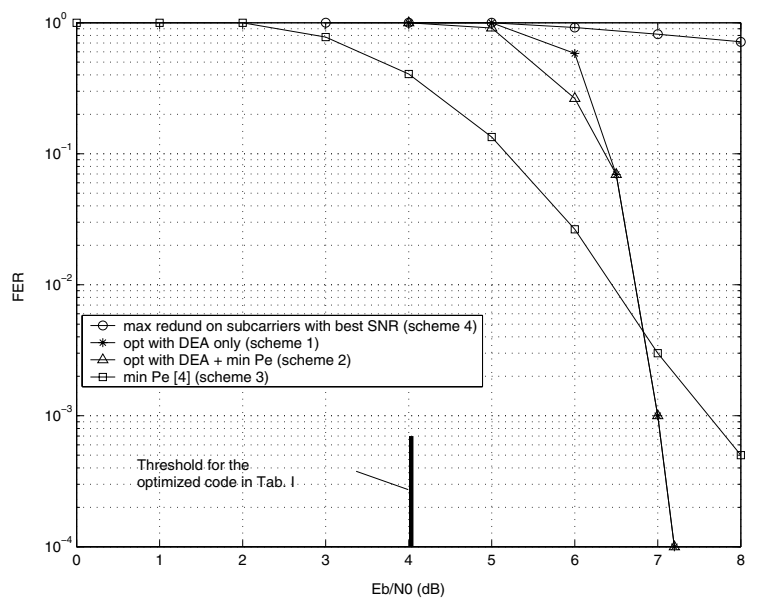

Fig. 4. Comparison between 4 strategies for Coded OFDM Transmission: 1) Code optimized by density evolution + optimal information bits positioning; 2) Code optimized by density evolution (positioning is not optimized), 3) Code optimized in [4], 4) Opposite strategy of the second case. The Frame Error Rate is plotted versus the average signal to noise ratio.

\section{CONCLUSION}

In this paper, we optimized the connectivity distribution of the LDPC code for OFDM transmission. The optimization of the irregularity has been done via the density evolution algorithm accordingly to the channel spectrum. We show that large gain particularly for low FER can be obtained for by exploiting the intrinsic unequal error protection of the irregular LDPC codes.

\section{REFERENCES}

[1] J.A.C. Bingham. Multicarrier modulation for data transmission: an idea whose time has come. Communications Magazine, IEEE, 28(5):5 - 14, May 1990.

[2] M. Karkooti and J.R. Cavallaro. Semi-Parallel Reconfigurable Architectures for Real-Time LDPC Decoding. In Proc IEEE International Conference on Information Technology (ITCC), volume 1, pages 579-585, April 2004.

[3] B. Lu, Yue Guosen, and Wang Xiaodong. Performance analysis and design optimization of LDPC-coded MIMO OFDM systems. Signal Processing, IEEE Transactions on [see also Acoustics, Speech, and Signal Processing, IEEE Transactions on], 52(2):348 - 361, Feb. 2004.

[4] V. Mannoni, D. Declerq, and G. Gelle. Optimized irregular gallager codes for ofdm transmission. In Personal, Indoor and Mobile Radio Communications, 2002. The 13th IEEE International Symposium on, volume 1, pages $222-226$, Sept. 2002.

[5] IEEE Std 802.16-2004 Revision of IEEE Std 802.16-2001. Ieee standard for local and metropolitan area networks part 16: Air interface for fixed broadband wireless access systems. Technical report, IEEE, 2004.

[6] T.J. Richardson, M.A. Shokrollahi, and R.L. Urbanke. Design of capacityapproaching irregular low-density parity-check codes. Information Theory, IEEE Transactions on, 47(2):619 - 637, Feb 2001.

[7] Sae-Young Chung, T.J. Richardson, and R.L. Urbanke. Analysis of sumproduct decoding of low-density parity-check codes using a gaussian approximation. Information Theory, IEEE Transactions on, 47(2):657 670, Feb 2001.

[8] S.J. Vaughan-Nichols. Achieving wireless broadband with WiMax. Computer, 37(6): 10 - 13, June 2004.

[9] W.Y. Zou and Wu Yiyan. COFDM: an overview. Broadcasting, IEEE Transactions on, 41(1):1 - 8, March 1995. 